THE SCATTER OF METALLICITIES OF STARS IN THE

\title{
SOLAR NEIGHBOURHOOD
}

\author{
B. FUCHS, C. DETTBARN AND R. WIELEN \\ Astronomisches Rechen-Institut Heidelberg, Germany
}

It is well known that the velocity dispersions of the stars in the solar neighbourhood increase with their ages (Wielen 1977). In Fig.1 we show $|W|$ weighted velocity dispersions (cf. Wielen 1977) of the stars in the Third Catalog of Nearby Stars (Gliese and Jahreiß 1994). Open symbols indicate main sequence stars and crosses indicate McCormick stars, a kinematically unbiased subset of the CNS3, respectively, whereas the filled symbols are the Edvardsson et al. (1993) data. Stars older than 14 Gyr are not shown because they are probably thick disk stars (Freeman 1991). We have assumed a maximum age of the old thin disk stars of 12 Gyr as suggested by the Edvardsson et al. data. As can be seen from Fig.1 both data sets fit ideally together. The solid line indicates a $\sigma \propto \tau^{1 / 2}$ law.

It is straightforward to derive from the epicyclic theory of stellar orbits the spatial dispersions corresponding to the velocity dispersions. For instance, the rms radial deviation of a stellar orbit from its original orbit is given by $\sigma_{R}=\sqrt{2.4} \sigma_{U} / \kappa$, if the galactic constants of the solar neighbourhood are used. $\sigma_{U}$ is the dispersion of the radial velocity and $\kappa$ is the epicyclic frequency, $\kappa=36 \mathrm{~km} \mathrm{~s}^{-1} \mathrm{kpc}^{-1}$.

If there is a galactic metallicity gradient it is quite obvious that the scatter of the metallicities of stars at a certain galactocentric distance, i.e. the solar neighbourhood, increases with the ages of the stars. Because older stars orbit on larger epicycles they can reach the solar neighbourhood from more distant parts of the galactic disk than younger stars. In Fig.2 we show metallicity dispersions determined from the Edvardsson et al. data versus radial dispersions calculated as described above. For this purpose the stars have been grouped into 5 age bins with about equal numbers of stars per bin. The mean ages of the groups are given in Gyr in Fig.2. The diagonal corresponds to a metallicity gradient of $-0.1 \mathrm{dex} / \mathrm{kpc}$. As can be seen from Fig. 2 there is a very close correlation between the predicted and 

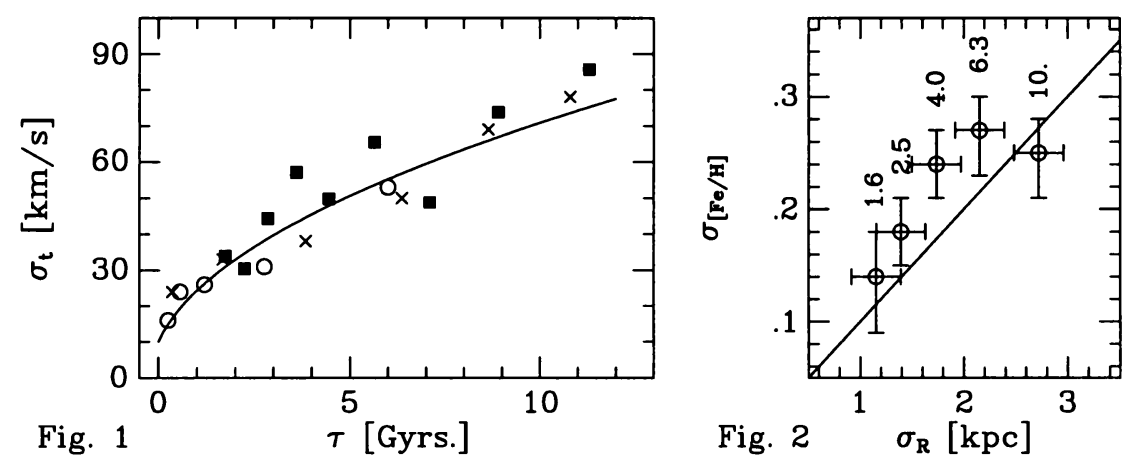

actually observed metallicity spread. The deviations are of order of 0.1 dex which we interpret as the inherent cosmic dispersion of the star formation processes. Such a reduced scatter of $[\mathrm{Fe} / \mathrm{H}]$ is expected in view of the low $[\alpha / \mathrm{Fe}]$ scatter of the stars (cf. contributions of P. Nissen and R. Wyse at IAU Symp. No.164).

François and Matteucci (1993) have drawn the same conclusions concerning the role of orbital diffusion as major source of the observed $[\mathrm{Fe} / \mathrm{H}]$ scatter. But Edvardsson et al. have put forward objections against this conjecture. They have considered a subset of stars in their sample which appear to lie on nearly circular orbits, and find that the metallicity scatter is essentially the same as for their full sample with no restrictions on the orbital parameters. They argue that these stars have always been close to the solar annulus so that the effect of orbital diffusion has been removed from the subset and that the large metallicity spread must be due to other effects. However the argument does not hold because stars which lie today on nearly circular orbits might have been scattered very recently onto them. We have derived elsewhere (Fuchs and Wielen 1987) the conditional probabilities for such cases and find that, looking backwards, the radial dispersion grows with age $\tau$ as $\sigma_{R}^{2}=D_{R} \tau$. We have determined the diffusion coefficient $D_{R}$ quantitatively and conclude that even in the restricted subset most of the observed metallicity scatter is due to orbital diffusion.

\section{References}

Edvardsson, B., Andersen, J., Gustafsson, B., Lambert, D.L., Nissen, P.E., Tomkin, J., 1993, $A \& A 275,101$.

François, P., Matteucci, F., 1993, $A \mathscr{E} A$ 280, 136.

Freeman, K.C. , 1991, in Dynamics of Disc Galaxies, B. Sundelius (ed.), Gőteborg, Sweden, p.15.

Fuchs, B., Wielen, R., 1987, in The Galaxy, G.Gilmore, B. Carswell (eds.), Reidel, p.375.

Gliese, W., Jahreiß, H., in preparation.

Wielen, R., 1977, $A \mathscr{E A} 60,263$. 